\title{
Isolated Non-Compaction Left Ventricle in Afrocaribbean \\ S Williams-Phillips
}

\begin{abstract}
Non-compaction of the left ventricle is a rare newly diagnosed cardiomyopathy (Grant 1926), and a significant cause of end stage triad of arrhythmias, heart failure, and systemic emboli. Twenty percent $(20 \%)$ having ventricular tachycardia leading to sudden death being one of the presenting signs and two-thirds having supraventricular arrhythmias. The familial forms can be X-linked, Autosomal Dominant or Autosomal recessive, with a variable genotype-phenotypic expression and sporadic mutations in sarcomere protein genes which are similar to hypertrophic, dilated and restrictive cardiomyopathy. The index case presented with increasing palpitations, heart failure at 21 years of age. This is the $1^{\text {st }}$ Case of isolated non-compaction of the left ventricle documented in an Afro-Caribbean.
\end{abstract}

Keywords: Cardiomyopathy, emboli, familial, heart failure, ventricular tachycardia From: TAI Wing, Andrews Memorial Hospital, Kingston, Jamaica, West Indies.

Correspondence: Dr S Williams-Phillips, Andrews Memorial Hospital, TAI Wing, 27 Hope Road, Kingston, Jamaica. West Indies. E-mail: sandrap@cwjamaica.com. 


\section{INTRODUCTION}

Left ventricle non-compaction (LVNC) is a rare structural cardiac anomaly characterized by spongy appearance of the myocardium was first described in autopsy specimens by Grant et al in $1926(1,2)$. Chin et al (3) described this entity as "isolated non-compaction of left ventricle myocardium" (ILVNC) although Zurich's Engberding first published the isolated rare entity ILVNC, in the absence of other types of structural heart disease, with embryonic prominent trabeculae spongy myocardial structures $(3,4)$. The aetiology of non-compaction is believed to be due to an arrest at eight weeks of foetal life, when the development of the vascularisation of the myocardium by the epicardial coronary arteries occurs. There is re-arrangement of trabeculae of the myocardium, starting from the epicardium, the base of the heart and interventricular septum to the free wall, ending in the apex of the left ventricle which is always involved. The congenital theory is contradicted by evidence of serial echocardiograms of confirmed ILVNC, where it was not evident in earlier studies (5).

ILVNC can be Autosomal dominant, X-linked and Autosomal recessive or a spontaneous mutation. The X-linked are more common in the paediatric population with mutations in G4.5 gene. The Adult population did not have the G4.5 gene, and were Autosomal dominant with the notation that the genetic cases in adults are distinct from the X-linked in children. The high rate of transmission in families of 18 to $50 \%$ provides need and justification for investigation of first degree relatives $(5,6)$.

The American Heart Association is the only group that classifies ILVNC as a separate primary genetic cardiomyopathy (6). There is no specific genotype- phenotype correlation with the same genetic mutations causes non-compaction in Noonan syndrome, Barth syndrome. Some types of congenital heart disease such as Aortic stenosis, Pulmonary atresia, Ebsteins anomaly and 
ventricular septal defects may have left ventricle non-compaction (LVNC) and the different phenotypic expression of seven genotypes with sarcoma protein gene, mitochondrial, cytoskeletal genes, led the European Society of Cardiology (7) and the 1995 World Health Organization (WHO) International Society and Federation of Cardiology Task Force to include ILVNC as an "unclassified cardiomyopathy"(8). The genetic heterogeneity and findings of ILVNC is also seen in dilated, hypertrophic and restrictive cardiomyopathies and is seen in wide variations of phenotypic expression of families suggests that ILVNC may not be a distinct entity $(5,7,8)$.

The clinical presentation of ILVNC ranges from the asymptomatic found during family screening with clinical symptoms and signs of arrhythmias, chest pain, heart failure with systolic and or diastolic dysfunction or signs of embolization and sudden death. The end stage of ILVNC includes the triad of arrhythmias, heart failure, and systemic emboli, with $20 \%$ having ventricular tachycardia leading to sudden death being one of the presenting signs and two-thirds having supraventricular arrhythmias (5-8).

Echocardiography is the main and primary diagnostic modality for ILVNC. The accepted ratio of endocardial non-compacted myocardium to outer epicardial compacted myocardium is 1.29 plus or minus 0.4. Adjunctive confirmatory investigations include Cardiac magnetic resonance imaging (CMRI), Tissue Doppler imaging, strain and rate and speckle tracking. The normal left ventricle twist of clockwise at the base and counter clockwise at the apex is not seen in ILVNC, where the base and apex rotate in the same direction. The presence of the endocardial non-compacted area being at least twice as thick as the epicardial compacted left ventricle is needed for diagnosis. Family studies with confirmed diagnosis of asymptomatic and symptomatic ILVNC show that the ratio may be less than the 2:1 accepted value. 
There is no unanimity of the Echocardiographic diagnostic criteria for ILVNC, as Chin et al uses the short axis view and Stollberger uses the apical four-chambered atypical views, to get the best image $(3,5,9)$. Chin et al (3), Peterson et al (10) and Jacquier et al (11), uses ventricular measurements at end diastole, Jenni et al uses the short axis view at end-systole (12). There have been revisions of the diagnostic Echocardiographic directives over the past two decades. The other diagnostic modalities include genetic testing, family screening of first-degree relatives, Electrophysiology studies and neurological assessment if there is suspicion of skeletal and mitochondrial myopathy $(5,12-15)$.

\section{CASE REPORT}

The Index case is an 21 years old Afro-Caribbean female in a tertiary level medical institution with a 2 year history of intermittent palpitations followed by shortness of breath and chest pain associated with dizziness which became more noticeable, significant and frequent 4 to 10 times daily with duration of 1 to 30 minutes. She also had intermittent pedal oedema for 2 years aggravated when standing for extended periods. She functions at NYHA1 but becomes NYHA 11 on with palpitations and on exertion. There was no history of syncope, seizure or deafness.

There was also no history of caffeine ingestion, energy drinks, high dose steroids, stimulants or illicit drugs. There is no family history of deafness, palpitations or use of pacemakers. On examination her weight was $63.4 \mathrm{~kg}$ and Height $165 \mathrm{cms}$ with BMI of 23.3 , and BSA $1.5 \mathrm{~m} 2$.

Cardiovascular examination revealed heart rate of 92 beats per minute with normal volume and rhythm. Her apex beat was at the 5th left intercostal space one centimetre outside the midclavicular line, normal in character. First and second heart sounds were normal. There were no 
cardiac murmurs. There were signs of cardiac failure. There was no pulmonary hypertension. Following one week of anti-arrhythmic and anti-failure therapy with complete cessation of symptoms and signs, the heart rate was 68 beats per minute, the apex was noted to be in the normal 5th left intercostal space in midclavicular line. Investigations revealed haemoglobin of $13.7 \mathrm{~g} / \mathrm{dl}$ and packed cell volume of $42.0 \%$. There were normal thyroid function tests.

Transthoracic echocardiogram showed in short-axis view at end systole, Figure 1, noncompacted interior left ventricular wall of $10.4 \mathrm{~mm}$ and compacted wall $4.1 \mathrm{~mm}$ with a ratio of 2.6:1. Normal fractional shortening of $50 \%$ and ejection fraction of $83 \%$. Colour Doppler, Figure 2, showed flow in deep trabeculations in interventricular septum, anterior, lateral and apical wall. There was trivial mitral and tricuspid regurgitation and normal right ventricular and pulmonary artery pressures. Normal origin and size of coronary arteries ruling out anomalous coronary arteries. Cardiac MRI is not available in Index country.

The resting electrocardiogram (ECG) showed heart rate of 90bpm, Sinus rhythm, and normal PR interval 150ms and QRS duration of 92ms. Normal QTc of $0.406 \mathrm{~ms}$ and QRS axis of 85. $\mathrm{P}$ axis was 79 and $\mathrm{T}$ axis was $67 . \mathrm{T}$ wave was inverted in V1 and $\mathrm{V} 2$ only. Intermittent isolated wide QRS premature ventricular contractions of 396ms were noted.

There was no pre-excitation syndromes eg Wolf- Parkinson- White, Long Ganong Levine, Mahaim, Ion Channelopathy, no Epsilon wave suggestive of Arrhythmoginic Ventricular Dysplasis, saddle back deformity ruling out Brugada syndrome or Takotsubo Cardiomyopathy. The ECG was not of any diagnostic value except for exclusion of specific cardiac electrophysiological disorders.

Medications initially started were cost effective anti-arrhythmic, anti-failure therapy, betablocker and diuretics. A dual chambered implantable cardio vertex defibrillator is recommended 
for insertion. There continues to be exercise restrictions with recreational activities, no competitive sports or sustained exertion.

\section{DISCUSSION}

ILVNC is a genetically- phenotypically heterogeneous disorder with no curative treatment. Despite the controversy with classification, genetic-phenotype expression and diagnostic criteria, the therapeutic strategies are currently symptomatic and aims to prevent sudden death and the classical end stage triad of arrhythmias, heart failure and systemic embolization. Treatment is directed at Heart failure therapy using guidelines for same, following via echocardiography, the asymptomatic left ventricular function and size, antiarrhythmic medications, anticoagulation for left ventricular ejection fraction (LVEF) below 40\% (5-8).

For secondary prevention of arrhythmias, implantable cardioverter/ defibrillator with its use for Primary use being a controversial issue. Biventricular pacing for advanced heart failure with syncope, ventricular arrhythmias and severely impaired LVEF less than 35\% with dysnchrony using guidelines. The timing and use of anti-coagulation is a controversial issue. Those with embolic events in earlier studies independent of ventricular function, and those with LVEF less than or equal to $40 \%$, anti-coagulation was used. ILVNC patients in sinus rhythm and with normal LVEF with or without medication, are a group still being debated, as it is thought that the deep trabeculations can be a nidus for thrombus formation (5-7).

This new entity ILVNC requires further prospective studies to ascertain true prevalence which is not known, and was diagnosed in symptomatic patients at 0.014 to $1.3 \%$ prevalence. Retrospective studies in Australia LVNC was 9.2\%, the third most common cardiomyopathy, 
which is similar to USA Texas hospital database of $9.5 \%$, with only $3 \%$ having ILVNC. The lack of unanimity of diagnostic, morphologic and echocardiographic views used to diagnose ILVNC show the limitations of echocardiographic criteria (5-7). Kohli et al found only $30 \%$ of patients satisfied all the diagnostic criteria of ILVNC by Stollberger et al, Chin et al, Peterson et al and Jacquiler et al $(3,9,10,11,13-15)$. He noted that 8 apparently healthy individuals satisfied the criteria for LVNC with half of this group being black. The current debate in this group speculate if there is an increased incidence in the black population or oversensitivity in diagnosing in this group and requires prospective assessment of this group of which this Index case belongs (3, 9$11,14,15)$.

The Index family would benefit from Genetic testing to determine if the gene responsible belong to any of the 7 phenotypes identified in ILVNC thus far, or if there is a different gene in the Afro-Caribbean population. An index case of Isolated Non-Compaction of the left ventricle documented for the first time in an Afro-Caribbean, in the English Medical Literature.

\section{REFERENCES}

1. Grant RT. An unusual anomaly of the coronary vessels in the malformed heart of a child. Heart 1926; 13: 273-83 
2. Freedom RM, Yoo SJ, Perrin D, Petersen, Anderson RH. The morphological spectrum of ventricular noncompaction. Cardiol Young 2005; 15: 345-64.

3. Chin TK, Perloff JK, Williams RG, Mohmann R. Isolated noncompaction of left ventricle myocardium. A study of eight cases. Circ 1990; 82: 507-13.

4. Engberg R, Bender F. Identification of a rare congenital anomaly of the myocardium by two-dimensional echocardiography: persistence of isolated myocardial sinusoids. Am J Cardiol1984; 53: 1733-34.

5. Oechslin E, Jenni R. Left ventricular non-compaction revisited: a distinct phenotype with genetic heterogeneity? European Heart J 31 Jan 2011 DOI: http://dx.doi.org/10.1093/eurheart/ehq508 1556-1456

6. Maron BJ, Towbin JA, Antzelevitch C, Corrado D, Arnett D et al. Contemporary definitions ad classification of the Cardiomyopathies: an American Heart Association Scientific Statement from the Council on Clinical Cardiology, Heart Failure and Transplantation Committee: Quality of care and Outcomes Research and Functional Genomics and Translational Biology Interdisciplinary Working Groups; and Council on Edidemiology and Preventiona. Circ 2006; 113: 1807-16.

7. Elliott P, Andersson B, Arbustimi E, Billinska Z, Cecchi F, Charron P et al. Classification of the cardiomyopathies: a position statement from the European Society Of Cardiology Working Group on Myocardial and Pericardial Diseases. Eur Heart J 2008; 29: 270-6

8. Richardson P, McKenna W, Bristow M, Malsch B, Mautner B, O’Connell J et al. Report of the 1995 World Health organisation/ International Society and Federation of Cardiology Task Force on the Definition and Classification of cardiomyopathies. Virc 1996; 93: 841842. 
9. Stolberger C, Finsterer J, Blazek G. Left ventricular hypertrabeculation/ noncompaction and association with additional cardiac abnormalities and neuromuscular disorders. Am J Cardiol 2002; 90: 899-902

10. Petersen SE, Selvanayagam JB, Westmann F, Robson MD, Francis JM, Anderson RH et al. Left ventricular non-compaction: Insights from cardiovascular magnetic resonance imaging. J Am Coll Cardiol 2005; 46: 101-5.

11. Jacquier A, Thuny F, Jop B, Giorgi R, Cohen F, Gaubert JY et al. Measurement of trabeculated left ventricle mass using cardiac magnetic resonance imaging in the diagnosis of left ventricle non-compaction. Eur Heart J 2010; 31: 1098: 104.

12. Jenni R, Oechslin E, Schneider J, Attenhoffer Jost C, Kaufmann PA. Echocardiographic and pathoanatomical characteristics of isolated left ventricular non-compaction: a step towards classification as a distinct cardiomyopathy. Heart 2001; 86: 666-71.

13. Kohli SK, Pantazis AA, Shah JS, Adeyemi B, Jackson G, McKenna WJ et al. Diagnosis of left-ventricular non-compaction in patients with left ventricular systolic dysfunction: time for a reappraisal of diagnostic criteria. Eur Heat J 2008; 29: 69-95.

14. Botto LD, Marino B. Left Ventricular noncompaction. Orphanet Encyc 2004:1-6.

15. Welford BC, Subbarao VD, Mulhern KM. Noncompaction of the left ventricular myocardium. Circ 2004; 109: 2965-71. 


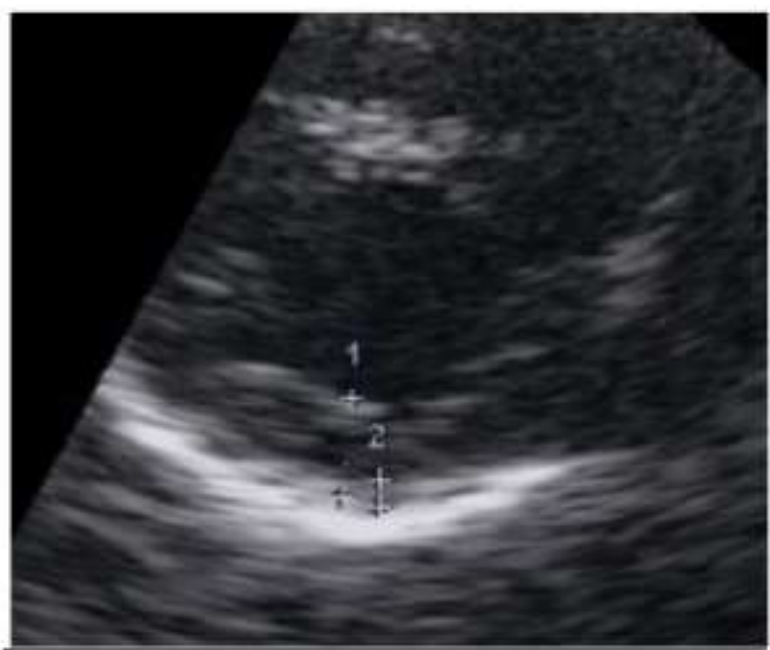

Fig 1. 2D image showing noncompacted left ventricle

Fig. 1. 2D image showing non-compacted left ventricle.

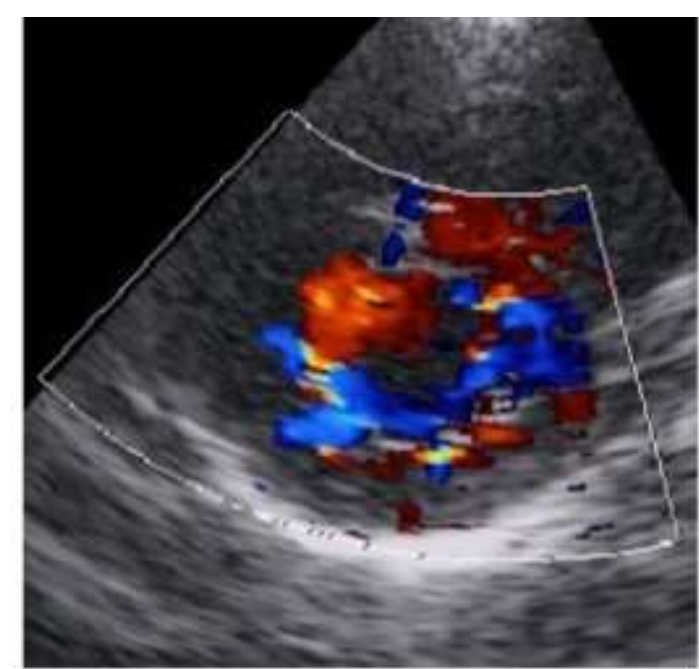

Fig 2. Color Doppler showing flow into non-compacted left ventricle

Fig. 2. Colour Doppler showing flow into non--compacted left ventricle. 